illumination is dark, indicating polypoid degeneration of the sinus-lining membrane, and above all when pus is present, it is advisable to open the antrum through the canine fossa.

These simple growths were up to quite recent years confused with the more formidable angiofibromas. In 1887 Sands, writing in the New Fork Medical Journal, removed a smooth mass pendent from the posterior nares with the galvano-cautery and remarked that very few are suitable for this method, as they are mostly attached to the skull by a broad base. In 1904 Fischer, a Belgian laryngologist, removed a pedunculated growth the size of a hen's egg from the nasopharynx of a boy aged 16 years, by means of a Gottstein's curette. In order completely to destroy the insertion, the nasopharynx was thoroughly curetted ; the slender pedicle appeared to spring from the left choana. These two cases were doubtless antro-choanal polypi. It was not till the year 1906 that Killian wrote an article on the origin of Mucous Polypi of the Choana and demonstrated that they originated in the antrum.

\section{POSTURAL SUBSARTORIAL BURSITIS.}

By R. Davies-Colley, M.Ch. Camb., F.R.C.S. Eyg., SURGEON, GUY'S HOSPITAL, LONDON.

Mr object in this communication is to draw attention to a form: of postural bursitis which has, as far as I am aware, never before been described. I had more than once met with cases of chronic inflammation and enlargement of the subsartorial bursa for which I could not account, before I discovered its very simple causation and its equally simple and most effective treatment. It was, in fact, only when I developed the condition myself and was puzzled to know why the swelling of the bursa and the accompanying pain were always greatest when I first stretched my legs in the morning, that the explanation suggested itself that it was indeed the night's rest that was the cause of the lesion. It is simple enough ; if one lies on one's side in bed with one knee resting upon the other, the inner tuberosities of the two tibiæ with the overlying tendons of the sartorius muscles and their burse are pressed directly against one another. It is, I believe, nothing more than this pressure that is the cause of the bursitis. It is, at any rate, a fact that if the pressure is taken off the tuberosities by one means or another the inflammation of the bursa rapidly subsides and does not return. I have met with some ten or a dozen cases during the last few years, about equally divided between the sexes.

Symptoms.-The symptoms are characteristic. They are: (a) Pain in the region of the inner tuberosity of the tibia on movement, especially marked during the first hour or two after rising in the morning. The pain develops gradually and without apparent cause : at first it may amount to no more than a mere stiffness which passes off during the day but later, when the bursa swells, it canses much discomfort and lameness. (b) Swelling over the tibial attachment of the sartorius muscle. This may be limited to the bursa, which becomes definitely outlined and can be shown to fluctuate. The amount of the swelling is seldom very great, though in one of my cases the sac was as large as a pigeon's egg and quite tense. At other times the swelling is diffuse, and there is inflammatory odema of the subcutaneous tissue over a considerable area as well as enlargement of the bursa. In these cases the skin is red and more or less acutely tender.

Diagnosis.-The diagnosis ought to present no difficulty, though every case referred to me has arrived with an erroneous one. The mistake has usually been to attribute the symptoms to disease of the head of the tibia or of the knee-joint, and chronic periostitis, abscess, and exostosis of the former and internal derangement, early tuberculous disease, osteoarthritis, and Morant Baker's cyst of the latter have all, at different times, been held responsible for them. The definite localisation of the pain and tenderness to the position of the bursa, the absence of excess of fluid in, and the perfect freedom of movement of the joint, and the lack of any history of trauma should be enough to exclude disease of the knee. The differential diagnosis from lesions of the bone may not be so easy, especially as an inflamed bursa is not uncommon over an exostosis in this situation, but if there is any doubt a radiogram will at once clear it up.
Treatment.-If the cause of the bursitis is borne in mind the treatment will be obvious. Fither the patient must be induced to sleep in such a position that the knees do not rest upon one another, or, better, a thick pad of wool should be worn at night over the inflamed bursa, so that if the knees do come into contact the pressure will be distributed, and the tuberosities of the tibiæ will not impinge directly upon each other. It is quite unnecessary to wear the pad during the day.

In all my cases the latter method of treatment has been rapidly and completely successful.

\section{DIFFERENTIAL DIAGNOSIS BETWEEN} CHOLELITHIASIS AND DUODENAL ULCER.

\section{By W. J. MCKEaNd, M.B., Ch.B. EdiN.}

THE following case presents certain features of interest which illustrate the difficulties sometimes met with in differentiating between duodenal ulcer and gall-stones.

History.-Mrs. X., aged 48, married, consulted me some months ago for epigastric pain and "indigestion." The pain apparently came on one to two hours after food, and she herself described it as a "hungry pain" because she soon found that the taking of food relieved it. It became more intense in the early hours of the morning, and she always had a plate of biscuits or light food by the bedside to alleviate it. She described it as being in the centre of the stomach, sometimes going right round like a girdle or straight through to the back. She had no sickness, but frequently a feeling of nausea, particularly when the pain was intense, and at these times she complained of a sour, acid taste in the mouth. Her past history was uneventful, except that she had suffered from occasional attacks of "indigestion" since her youth and had always been constipated.

Examination.-There was tenderness over the epigastrium and right hypochondrium, its maximum point of intensity being just to the right of the middle line. She had tenderness behind about the level of the tenth dorsal. Skin sensitiveness was much more pronounced to the right of the middle line. Murphy's sign-tenderness on pressure over the tip of the ninth costal cartilage on deep inspiration in the sitting position-was absent. No enlargement of liver or gallbladder could be made out. There was no jaundice and the other svistems were negative. Nothing abnormal was found in the urine. A test-meal was suggested, but on explaining its terhnique was refused.

Treatment.-A specimen of fæces was sent to a pathologist and the presence of occult blood detected. A diagnosis of duodenal ulcer was felt to be justified, and she was treated on this hypothesis. She was put to bed at rest for six weeks, carefully dieted, and treated on the usual medical lines. Her condition improved, the pain, except for occasional attacks during the night, was very much better, and she found she could take a reasonable amount of food without discomfort. She was sent to the south of France for three months; whilst there she wrote and told me that so long as she stuck to her diet she felt very well, but if she attempted to take any luxuries she had a recurrence of the pain. She remained comparatively well for six to eight weeks on her return to London, and then her symptoms recurred as bad as ever. She was again rested and dieted, and again improved. This improvement was not maintained, however, and the nocturnal pain became more severe. An operation was advised, but before submitting to it she thought she would like another opinion. She saw a consulting physician, who confirmed the diagnosis of duodenal ulcer and advised rest and diet for six months. As her condition did not improve and she was becoming very neurotic I sent her to a radiologist for a barium meal. He reported as follows "The stomach is active and very irritable. There is irregularity of the duodenal cap, which is in favour of a diagnosis of duodenal ulcer. The colon is ptosed and shows marked stasis with a suggestion of adhesions between the ascending and the first part of the transverse colon."

I persuaded the patient to see a surgeon, and an operation was agreed to.

Operation.- At the operation, performed by Mr. L. E. Barrington-Ward, a distended gall-bladder full of stones was found, with slight congestion of the duodenum in its immediate vicinity. The gall-bladder was removed, and the patient made an uneventful recovery. Since her operation she has had no pain and "can eat anything."

\section{Remarks.}

The curious points about the case are, I think, the type of pain so characteristic of duodenal ulcer, the presence of occult blood in the stools, and the apparent beneficial effect of rest and diet, together with the 